\title{
The Effects of Textured Insoles on Balance in Individuals with Knee Osteoarthritis
}

\author{
Hyoungin Park \\ Department of Kinesiology, University of Georgia, USA
}

Copyright $\mathrm{C} 2018$ by authors, all rights reserved. Authors agree that this article remains permanently open access under the terms of the Creative Commons Attribution License 4.0 International License

\begin{abstract}
As evidence of the effectiveness of added plantar-surface texture to improve balance has been successful for various populations, it is important to study if textured insoles may increase balance for individuals with knee osteoarthritis (OA) who have compromised the somatosensory function of the affected joint and correspondingly an increased risk of falls. Thus, this study investigated the degree of benefit through the use of textured insoles for improving balance and compared the balance of individuals with knee OA to matched-healthy peers. Fifteen with knee OA and fifteen healthy, aged, gender and BMI matched controls completed this study and were evaluated on balance as measured by a computerized dynamic posturography. There were significant improvements in balance when wearing the textured insoles in both groups, and healthy knee controls showed significantly better balance performances than individuals with knee OA. The benefits of this study for the individuals with knee OA are that this may lead to the development of an evidence-based footwear intervention which is noninvasive, simple to use, inexpensive, allows the user for self-management, and has the capacity to reduce the risk of falls, consequentially improving the quality of life.
\end{abstract}

Keywords Knee Osteoarthritis, Balance, Textures Insoles, Computerized Dynamic Posturography

\section{Introduction}

Adults aged $45 \mathrm{yr}$ and older with arthritis account for $52 \%$ of adults in the United States [1]. Among the arthritis forms, osteoarthritis (OA) is the most prevalent and is a leading cause of disability and loss of function $[2,3]$. OA is characterized by a degradation of articular cartilage, sclerosis of the subchondral bone, and osteophyte formation with symptoms of joint pain and dysfunction [4]. In its advanced stages, joint contractures, muscle atrophy, limb deformity, and the prior characteristics combine to result in a reduction of lower limb muscle strength and abnormal somatosensory function $[5,6,7]$. As a result, individuals with knee OA have been reported to have balance deficits $[8,9,10]$, and knee OA is a major risk factor for falls [11]. Physical interventions to improve balance in individuals with knee OA have implemented various approaches such as aerobic and weight training, tai chi, aquatic exercise, kinesthesia balance exercises and sensorimotor training. However, no method of physical interventions has been universally agreed upon. Furthermore, although individuals with knee OA use many fall prevention tactics, they often fall more compared to those without arthritis [12]. Therefore, additional types of intervention strategies aimed at improving balance for individuals with knee OA should be considered.

The plantar sole is a unique body part because the plantar sole, which is densely packed with mechanoreceptors, is the primary location on the body directly connecting humans to their environment. It has been shown that plantar sole feedback can be used to detect information about support surface characteristics by providing information about pressure changes and associated body sway $[13,14]$. Bodies use this somatosensory information to initiate reflexes that help maintain balance $[15,16]$. Previous research has been conducted into how changing the quality of the somatosensory information received from plantar sole determines balance is affected. For example, ischemia of the foot, desensitization via anesthetizing or cooling of the plantar surface, was used to decrease or alter the somatosensory input from the foot and plantar soles, which revealed postural instability $[17,18]$. Thus, postural instability using desensitized/anesthetized feet emphasizes the importance of somatosensory feedback in maintaining balance. Naturally, a number of studies have been conducted investigating how enhancing somatosensory feedback can improve balance. Specifically, the ability to enhance cutaneous information on the feet has been reported to be successful. Orth et al. [19] (2013) showed previous studies in a systematic review that the stimulation of sensory receptors in the skin via simple 
mechanical deformation of the plantar surface by added texture (e.g., addition of nodules and protuberances on the surface of a shoe insole or a standing area) can improve balance. Evidence of the effectiveness of added plantar-surface texture has been demonstrated in various populations, such as the young, the elderly, fallers, people with Parkinson's disease, people with multiple sclerosis, and people with chronic ankle instability under static balance test, dynamic balance test, gait analysis and proprioception test [19]. For example, Qiu et al. [20] (2012) concluded that textured material could improve balance in older people in unstable surface conditions. These improvements were strongest under conditions where reliance on somatosensory information was emphasized by removal of visual information, which indicated that the somatosensory information received from the plantar surface is critical for maintaining balance. In this regard, it is apparent that textured material enhanced somatosensation in the skin of the plantar surface. Therefore, this present study has suggested that textured insoles may provide an effective intervention to decrease balance instability and can potentially enhance somatosensory feedback and ultimately improve the functionality of balance.

Based on previous research, knee OA may negatively impact balance. However, there are few studies that have evaluated the balance performance of individuals with knee OA using computerized dynamic posturography. Furthermore, it is unclear whether the enhanced somatosensory input provided by a textured insole would benefit an individual with knee OA compared to other previously studied populations. As individuals with knee OA have compromised the somatosensory function of the affected joint and correspondingly an increased risk of falls [7, 21], understanding the effects of textured insoles on balance of these individuals is crucial. Therefore, it is important to study how using textured insoles may improve balance for individuals with knee $\mathrm{OA}$ and if textured insoles may increase balance in healthy matched control individuals, though it may be less efficient for individuals who are physiologically normal. Thus, this study investigated the degree of benefit provided to these two populations through the use of textured insoles for improving balance and compared the balance of individuals with knee OA to matched-healthy peers.

\section{Methods}

\subsection{Participants}

Fifteen participants (12 female, 3 male) with knee OA and 15 asymptomatic controls (12 female, 3 male) aged between 32 and $62 \mathrm{yr}$ were recruited through advertisements in local clubs and university listserve. The study was approved by the university's Institutional Review Board, and written informed consent was obtained from all participants. Fifteen participants in each group were calculated via an a priori power analysis to provide $85 \%$ power with an effect size of approximately 1 at $\alpha=.05$ to detect differences. The data used for the estimates were the results of EQ scores reported in a study that was done by Jones and colleagues on postural control of individuals with fibromyalgia [39].

All participants were free from (1) a concomitant medical illness that, as judged by the principal investigator using medical history and health questionnaire, could impair balance (for example, neurological or significant musculoskeletal disease, Meniere's disease or other inner ear disease, permanent lower-limb injury, significant psychiatric disorder), (2) the inability to ambulate without an assistive device, (3) an abnormal optometric or ophthalmic examination in the 6 months. Diagnosis of knee OA was confirmed by rheumatologists who were each participant's physician. And pain level was measured by the WOMAC (Western Ontario and McMaster Universities Osteoarthritis Index).

Exclusion criteria include the following: inflammatory arthritis, major lower extremity joint surgery (e.g., knee arthrotomy within the previous 6 months), any condition which severely limits local ambulation (e.g., amputation or stroke), use of gait aids for ambulation, or dementia or inability to understand and follow directions. Participants with knee OA were matched with asymptomatic control participants based on gender, age, and body mass index (BMI). Control participants with evidence of rheumatoid or any other type of arthritis, a history of injury to the lower extremity, or prolonged knee pain that required medication and knee surgery were excluded. Control participants with recurring or prolonged knee pain occurring within the last month, even if pain-free on the day of testing, were excluded. Participant characteristics are presented in Table 1 .

Table 1. Participant Demographics

\begin{tabular}{|c|c|c|c|c|c|}
\hline $\begin{array}{c}\text { Participant } \\
\text { Characteristics }\end{array}$ & $\begin{array}{c}\text { Knee OA (n=15) } \\
\text { Mean (S.D.) }\end{array}$ & $\begin{array}{c}\text { Healthy knee }(n=15) \\
\text { Mean (S.D.) }\end{array}$ & $F$ & $P$ value & $\eta_{\mathrm{p}}{ }^{2}$ \\
\hline Age $(\mathrm{yr})$ & $52.67( \pm 11.36)$ & $51.40( \pm 10.78)$ & 0.098 & 0.756 & 0.003 \\
\hline Height $(\mathrm{cm})$ & $164.59( \pm 10.30)$ & $162.90( \pm 6.57)$ & 0.288 & 0.596 & 0.010 \\
\hline Mass $(\mathrm{kg})$ & $75.70( \pm 13.96)$ & $73.40( \pm 13.74)$ & 0.207 & 0.653 & 0.007 \\
\hline BMI $\left(\mathrm{kg} / \mathrm{m}^{2}\right)$ & $28.07( \pm 5.38)$ & $27.61( \pm 4.68)$ & 0.063 & 0.803 & 0.002 \\
\hline
\end{tabular}




\subsection{Materials}

The textured insoles (flexible, polyvinyl chloride [PVC], $3 \mathrm{~mm}$ thickness, transparent) had small, round peaks with center-to-center distances of approximately $4 \mathrm{~mm}$ (Figure 1). Insoles were customized for both left and right feet based on the participant's foot width and length. This textured insole was considered adequate to deliver sensory stimulation, but not rough enough to cause skin discomfort.

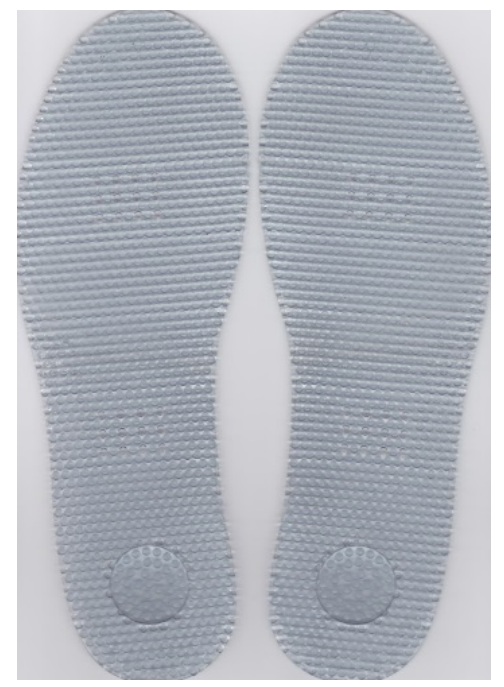

Figure 1. The textured insoles used in this study

\subsection{Procedures}

Upon acceptance into the study, each participant underwent warmups before practice testing. Warm-ups included dynamic stretches, such as hip circles, arm circles, arm swings and walking. The participant then practiced performing the two balance tests (sensory organization test [SOT], motor control test [MCT]) described below. The participant performed NeuroCom EquiTest SOT and MCT protocol with smooth insoles. There was a ten-minute rest period to change insoles. The participant then wore the textured insoles and was tested again.

\subsection{Sensory Organization Test (SOT), Equilibrium Score, and Composite Equilibrium Score}

The sensory organization test (SOT) challenges stability via sway referencing by altering the availability and/or accuracy of sensory information from three systems: the visual, vestibular, and somatosensory systems. Sway referencing synchronizes the rotation of the platform and/or surroundings with the person's anterior-posterior postural sway; that is, as the individual sways forward, the platform and/or surrounding room sways forward synchronously. The sway-referenced platform minimizes or alters the accuracy of somatosensory information, whereas the sway-referenced surrounding, extending beyond the visual periphery, provides inaccurate visual sensory information. By reducing the quality of sensory feedback regarding postural sway, these methods challenge postural control. Three trials for each sensory condition were presented in the manufacturer's suggested order, with increasing difficulty from condition 1 through condition 6 (Table 2).

Table 2. Description of the six sensory organization test tasks

\begin{tabular}{|c|c|c|c|c|}
\hline \multirow{2}{*}{ Condition } & \multicolumn{2}{|c|}{ Environment } & \multicolumn{2}{|c|}{ Expected Sensory System Response } \\
\hline & Vision & Surface & Removed and/or reduced & Using \\
\hline 1 & Eyes open & Fixed & & Somatosensory \\
\hline 2 & Eyes closed & Fixed & Vision & Somatosensory \\
\hline 3 & Sway reference-visual surrounding & Fixed & Vision & Somatosensory \\
\hline 4 & Eyes open & sway-referenced surface & Somatosensory & Vision \\
\hline 5 & Eyes open & sway-referenced surface & Somatosensory \& Vision & Vestibular \\
\hline 6 & Sway-referenced visual surrounding & sway-referenced surface & Somatosensory \& Vision & Vestibular \\
\hline
\end{tabular}


The equilibrium score (ES) indicates how well the participant's sway remains in the expected angular limits of stability during SOT trials. The ES was generated from forceplate data of each trial (20 seconds @ 100Hz, 2000 data points) via NeuroCom software (NeuroCom, Clackamas, OR). An ES was computed for each trial using the following equation:

$$
\mathrm{ES}=\frac{12.5-\left[\theta_{\max }-\theta_{\min }\right]}{12.5} * 100
$$

The result was provided as an inverse percentage of 0-100. While no movement resulted in an ES of 100, a fall resulted in a score of 0 . This outcome measure is clinically accepted and has been used extensively in motor control research $[22,23]$. The composite equilibrium score (CES) quantifies the weighted average of the scored of each condition. It was designed to measure the overall balance performance and can be used to predict the risk of falls [40].

\subsection{Motor Control Test (MCT) and Latency}

In the motor control test (MCT), the participant's automatic reactions were measured in response to support surface translations. The MCT consisted of 6 conditions: graded backward (3) and forward (3) translations (Figure $2)$. The translations were scaled according to the participant's height, but durations were the same for everyone. Small, medium and large translations produced a $1.25 \mathrm{~cm}$ translation for $250 \mathrm{~ms}, 3.14 \mathrm{~cm}$ translation for $300 \mathrm{~ms}$, and $5.7 \mathrm{~cm}$ translation for $400 \mathrm{~ms}$, respectively. Small translations represented threshold stimulation, large translations produced a maximal response, and medium translations were midway between the small and large. Each translation occurred at a constant velocity and therefore transferred constant forward or backward angular momentum to the participant's body [24].
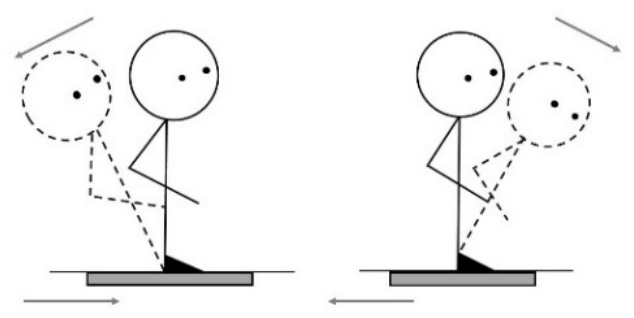

Figure 2. Forward/Backward Translations

The latency is defined as the time in milliseconds between the onset of translation during the MCT and the onset of the participants' response to the support surface translation movement. Latencies were the averaged performance of the right and left feet.

\subsection{Statistical Analysis}

Regression was performed to evaluate the interaction between groups to investigate if textured insoles produce greater improvements in knee OA groups. This analysis had two groups of subjects (knee OA and healthy knee groups) applied to the variables (difference scores between the smooth insole and the textured insole) of equilibrium scores and latency. The dependent $(\mathrm{Y})$ variable was the difference between smooth and textured insoles. The predictor variables $(\mathrm{X})$ was the existence of knee OA (grouping variable), with covariates such as participants' age and smooth insole scores. To find group interactions the following equation was used:

Y_Texrued insole - Y_Smooth insole $=$ intercept $+b 1$ * grouping variable (knee $\mathrm{OA}=1$ and healthy knee group $=0$ )

$$
+\mathrm{b} 2 * \text { age }+\mathrm{b} 3 * \text { Y_Smooth insole }+\mathrm{e}
$$

The age as one of the covariates was chosen based on empirical evidence that has shown deterioration of balance performance associated with aging [25].

Furthermore, paired $t$-tests were conducted to determine whether the textured insole improved balance performances in each group, and independent t-test was utilized to identify differences in the balance between the knee OA and healthy control group with smooth insole conditions. For the normality test, a Shapiro-Wilk analysis of the data was completed to determine if the assumption of normality had been met, and the Levene's test for equality of variances was utilized to assess homogeneity. Wilcoxon signed-rank test for paired $t$-test and Mann-Whitney $\mathrm{U}$ test for Independent $t$-test were used if the data violated normality test assumptions. The results of the evaluation of the significance of the differences were represented by the significance coefficient $(p)$. The level of statistical significance was indicated by “*” $-p<0.05$.

\section{Results}

\subsection{Interactions between Groups}

There were no statistically significant interactions between groups in regression analysis, indicating that the textured insoles had similar effects on all participants. 
Table 3. Results of coefficients

\begin{tabular}{|c|c|c|c|c|c|}
\hline & \multicolumn{2}{|c|}{ Unstandardized Coefficients } & \multirow{2}{*}{$\frac{\text { Standardized Coefficients }}{\beta}$} & \multirow{2}{*}{$\mathrm{T}$} & \multirow{2}{*}{ Sig. } \\
\hline & B & Std. Error & & & \\
\hline Condition 1 & -.904 & .649 & -.240 & -1.393 & .175 \\
\hline Condition 2 & -.341 & .834 & -.047 & -.410 & .685 \\
\hline Condition 3 & -.892 & 1.417 & -.103 & -.629 & .535 \\
\hline Condition 4 & -2.687 & 3.694 & -.123 & -.727 & .473 \\
\hline Condition 5 & 2.993 & 3.097 & .109 & .966 & .343 \\
\hline Condition 6 & 1.676 & 3.031 & .092 & .553 & .585 \\
\hline Composite equilibrium score & 0.962 & 1.875 & .080 & .513 & 612 \\
\hline Latency & -.477 & 1.777 & -.054 & -.268 & .790 \\
\hline
\end{tabular}

\subsection{Effects of Textured Insoles on Each Group}

The presence of the textured insole affected condition 6 and composite equilibrium score (CES) of both groups; the textured insole $(\mathrm{M}=60.578, \mathrm{SD}=16.084$, conditions; $\mathrm{t}(14)=-3.323, p=0.005)$ and the smooth insole $(\mathrm{M}=51.044$, $\mathrm{SD}$ $=19.449)$ in the knee $\mathrm{OA}$ group, and the textured insole $(\mathrm{M}=68.00, \mathrm{SD}=10.262$, conditions; $\mathrm{t}(14)=-2.446, p=0.028)$ and the smooth insole $(\mathrm{M}=64.289, \mathrm{SD}=11.160)$ in the healthy knee control group for condition 6 , and the textured insole $(\mathrm{M}=71.85, \mathrm{SD}=9.881$, conditions; $\mathrm{t}(14)=-3.590, p=0.003)$ and the smooth insole $(\mathrm{M}=64.74, \mathrm{SD}=13.136)$ in the knee OA group, and the textured insole $(\mathrm{M}=77.54, \mathrm{SD}=5.139$, conditions; $\mathrm{t}(14)=-3.405, p=0.004)$ and the smooth insole $(\mathrm{M}=75.24, \mathrm{SD}=6.009)$ in the healthy knee control group for the CES. Also, in condition 4 of the healthy knee group, because the data were not normally distributed, a Wilcoxon Signed-Ranks test was run, and the output indicated that the textured insole scores were statistically significantly higher than the smooth insole scores $(\mathrm{Z}=2.445, \mathrm{p}=0.014, \mathrm{r}$ $=0.45)$. In condition 5 , there were significantly higher scores for the textured insole $(M=53.734, S D=14.485$, conditions; $\mathrm{t}(14)=-4.039, \mathrm{p}=0.001, \mathrm{~d}=-1.043)$ compared to the smooth insole $(\mathrm{M}=37.022, \mathrm{SD}=25.674)$ in the knee OA group. In addition, there was not a significant difference in the latency for the textured insole condition in both groups

\subsection{Knee OA Group vs. Healthy Knee Group}

Independent t-tests (Table 4) and Mann-Whitney U tests (Table 5) were conducted to compare the smooth insole's equilibrium scores in individuals with knee OA and healthy knee controls. For the four conditions in which either the force plate and/or surround moved, and CES, the groups differed for the smooth insole. Healthy knee group had significantly higher scores than knee OA group. Also, an independent $t$-test indicated that latency of the smooth insole condition was faster for healthy knee controls $(\mathrm{M}=129.80, \mathrm{SD}=9.511)$ than for individuals with knee $\mathrm{OA}(\mathrm{M}=138.367$, $\mathrm{SD}=11.578, \mathrm{t}(28)=-2.214, p=0.035)$.

Table 4. Results of independent t-tests

\begin{tabular}{|c|c|c|c|c|c|c|}
\hline Task condition & Group & Mean & SD & Std. Error Mean & $t$ & $p$ \\
\hline \multirow{2}{*}{ Condition 1} & Health & 93.87 & 1.489 & 0.385 & \multirow{2}{*}{1.455} & \multirow{2}{*}{0.158} \\
\hline & knee OA & 92.84 & 2.278 & 0.588 & & \\
\hline \multirow{2}{*}{ Condition2 } & Health & 88.38 & 4.539 & 1.172 & \multirow{2}{*}{0.811} & \multirow{2}{*}{0.424} \\
\hline & knee OA & 87.02 & 4.608 & 1.19 & & \\
\hline \multirow{2}{*}{ Condition5 } & Health & 58.56 & 9.803 & 2.531 & \multirow{2}{*}{3.035} & \multirow{2}{*}{$0.007^{*}$} \\
\hline & knee OA & 37.02 & 25.674 & 6.629 & & \\
\hline \multirow{2}{*}{ Condition6 } & Health & 64.29 & 11.16 & 2.881 & \multirow{2}{*}{0.017} & \multirow{2}{*}{$0.032 *$} \\
\hline & knee OA & 51.04 & 19.449 & 5.022 & & \\
\hline \multirow{2}{*}{ CES } & Health & 75.24 & 6.009 & 1.552 & \multirow{2}{*}{0.002} & \multirow{2}{*}{$0.011 *$} \\
\hline & knee OA & 64.74 & 13.136 & 3.392 & & \\
\hline \multirow{2}{*}{ latency } & Health & 129.8 & 9.511 & 2.456 & \multirow{2}{*}{0.555} & \multirow{2}{*}{$0.035^{*}$} \\
\hline & knee OA & 138.37 & 11.578 & 2.99 & & \\
\hline
\end{tabular}


Table 5. Mann-Whitney U tests results

\begin{tabular}{|l|c|c|c|c|c|c|c|}
\hline & Mann-Whitney U & Wilcoxon W & Z & Sig. & Median (Healthy) & Median (Knee OA) & Effect Size $r$ \\
\hline Condition 3 & 61.500 & 181.500 & -2.118 & $0.033 *$ & 91.670 & 90.000 & -0.547 \\
\hline Condition 4 & 65.500 & 185.500 & -1.950 & $0.049 *$ & 78.330 & 69.670 & -0.503 \\
\hline
\end{tabular}

\section{Discussion}

\subsection{Interactions}

Although it was expected that a textured insole intervention would result in greater improvements in the knee OA group compared to the healthy group, there were no statistically significant interactions between groups, indicating that textured insoles had similar effects for individuals with knee $\mathrm{OA}$ and with healthy knees. However, regarding the results of the paired- $t$ test, there were small interactions between groups. This difference in results may be attributed to a small sample size or may result from the covariates only existing in the regression models and not in $t$-tests.

\subsection{Effects of Textured Insoles on Each Group}

Although there were no interactions, paired $t$-tests were conducted to find out whether the textured insoles improved the balance of each group. In the knee OA group, there were significant improvements in condition 5, 6, and CES when wearing the textured insoles. In the healthy knee control group, there were statistically significant improvements in condition 6 and CES when wearing the textured insoles.

Condition 6, where the visual and somatosensory information was altered, was the only condition where both groups were affected by textured insoles. All participants with the textured insoles were able to better manage balance in inaccurate visual and somatosensory situations, leading to a higher reliance on somatosensory orientation. This result may be due to hyperesthesia of the plantar surfaces of the feet, resulting in increased cutaneous afferent receptor activity while participants wore the textured insoles. Specifically, the underlying physiological mechanisms by which textured insoles can cause changes in the balance suggest that textured insoles provide enough stimulation to alter the discharge rate from mechanoreceptors, or firing patterns of sensory afferents, located in the skin of the plantar surface [26]. This effect would result in an overall increase of neural feedback from the cutaneous receptors to the central nervous system, which potentially contributed to improving balance. Also, this finding is consistent with previous work by Qui et al. [20] (2012), demonstrating that textured insoles improved balance further in a challenging situation where visual and somatosensory inputs were conflicted. However, it is important to note that their research did not attempt to add textured insoles into shoes but rather explored the effects of a textured standing surface in healthy older adults.

As CES score represents an overall balance performance, the textured insoles improved balance in the knee OA group most likely due to the enhancement of somatosensory information from the plantar surfaces. These results contribute to the current understanding of the research by complementing the extant data. For instance, our results are in agreement with data reported by Priplata et al. [27] (2002) who explored the effects of vibration insoles, showing that balance can be improved during quiet standing through enhanced somatosensory feedback. Additionally, much of previous research demonstrated that providing stimulation to the plantar surface of the feet through the vibration stimulation has been considered as a potential mechanism through which footwear intervention could improve balance $[28,29]$ by altering sensorimotor function. The difference between previous research and the current study was the characteristics of the stimulation to the feet. In this study, the plantar surface persisted in contact with the indentations of the textured insole. When the cutaneous afferents respond continuously to prolonged indentations, they are classified as slow-adapting mechanoreceptors [15]. In comparison, vibratory interventions could manipulate the frequency, intensity, phase, and duration of stimulation. These interventions may affect fast-adapting mechanoreceptors which show burst responses to stimulation [30]. Textured insoles used for this study did not work on the same principle because these textured insoles did not provide electrical stimulation.

In addition, such vibration devices may be expensive and difficult to use as effective interventions to improve balance in daily life. Textured insoles may provide a practical alternative and act as an inexpensive way of improving balance [31]. A systematic review conducted for the effects of textured interventions demonstrated that the stimulation of sensory receptors in the skin through simple mechanical deformation of the plantar surface by added texture could improve the balance performances in various populations, and could lead to an increase in proprioceptive sensitivity and a higher reliance on the somatosensory information [31]. Although we do not know the structural integrity or functional capabilities of the sensory receptors in the superficial plantar tissues in the individuals with knee OA, it is surmised that textured insoles can successfully stimulate sensory receptors to increase their output, as known to occur in healthy adults and other clinical populations, and thereby improve balance.

Also, the habituation of participants to the textured insoles is one of the critical aspects of future research 
needed in this area. In Palluel, Olivier, \& Nougier [32] (2009) study, the participants were instructed to stand or to walk for 5 minutes with textured insoles. However, effects of textured insoles in supporting perceptual-motor function need to be studied over a much longer period spanning several months [31]. Although our participants testified that the textured insoles were comfortable during the assessments, it is important to assess over extended periods of time to ensure the long-term adherence will be comforted.

\subsection{Knee OA Group vs. Healthy Knee Group}

The results of the balance performances with smooth insoles between the individuals with knee $\mathrm{OA}$ and the healthy knee controls showed that condition 3, 4, 5, 6, and CES were significantly higher for healthy knee controls than individuals with knee OA. Lower EQ values for knee OA group were evidence of larger displacements of the center of gravity in the forward-backward direction. CES score showed an overall performance level of both group, and a lower score suggests that the OA group was unable to maintain balance and a stable position during the SOT test procedure compared to healthy knee group. In condition 5 and 6 tests where participants had to compensate for the visual deprivation and the inaccurate somatosensory information with an increased use of vestibular information, there were the increased number of falls and dropped equilibrium scores. However, there were small differences in conditions that did not stress balance. Several potential mechanisms may account for the balance deficit observed in the OA group, although this cross - sectional study does not allow these to be confirmed. Individuals with knee OA often exhibit several factors that affect the balance negatively, including muscle weakness [33], impaired somatosensory [7], a significant decline of mechanoreceptors compared to age-matched healthy peers [5], and joint contracture [34].

In addition, individuals with knee OA displayed a longer neuromuscular response latency to balance perturbations, indicating a reduced ability to begin in recover balance quickly following an unexpected disturbance. The previous research has stated that this long latency response could also result from the lack of dependence on somatosensory and vestibular information [35] which are used to activate and modulate balance correcting responses [36]. Joint pain associated with the knee OA may play a role in slower latency as well. Joint pain changes the responses and affects the muscle activity during the automatic response [37]. All knee OA participants of this study had mild-moderate knee joint pain when they were assessed. Experimentally-induced thigh pain results in larger sway area, increased sway displacement, increased electromyographic (EMG) activity, and increased time to return to an equilibrium position after unexpected perturbation [38].
These observations should be taken into account in the fall prevention, especially, participants should place in dynamic situations with the conflicted sensory environment, as these are found in daily life. Also, The current study has some limitations. First, the criteria for diagnosis of knee OA may be different depending on each participant's physician due to the fact that the diagnosis of knee OA is based on several factors, including the individual's age, history, and symptoms. This may have caused participants with asymptomatic osteoarthritis to be placed in the control group.

Second, due to the lengthy test procedures, the author did not want to risk fatigue and habituation of the textured insoles in this first study with individuals with knee OA and, therefore, used one trial with practice trials rather than multiple trials for balance tests. Although this might have resulted in measurement error in the data, it seems that the magnitude of the textured insole effect on balance was larger than the measurement error which suggests this was a true effect of the textured insoles. Also, there was no period of accommodation and habituation regarding the textured insoles. Although our participants testified that the textured insoles were comfortable during the tests, it is important to assess comfort levels over extended periods of time to ensure the long-term adherence will be comforted and that there will be no habituation effects as we did not investigate the effects of prolonged exposure to the textured insoles.

\section{Conclusions}

The results indicate that a textured insole intervention can induce positive changes in balance overall. The benefits of this study for the individuals with knee OA are that this may lead to the development of an evidence-based footwear intervention which is noninvasive, simple to use, inexpensive, allows the user for self-management, and can reduce the risk of falls, consequentially improving the quality of life. We believe that this study has clinical significance based on the fact that falling is one of the leading causes of injury in the knee OA population. Future studies which examine effects of prolonged wearing textured insoles are needed to conclude if a textured insole intervention can produce significant changes in balance and function in knee OA population.

\section{REFERENCES}

[1] CDC. (2013). Prevalence of doctor-diagnosed arthritis and arthritis-attributable activity limitation - United States, 2010-2012. MMWR: Morbidity \& Mortality Weekly Report, 62(44), 869-873.

[2] CDC. (2001). Prevalence of disabilities and associated health conditions among adults -- United States, 1999. 
MMWR: Morbidity \& Mortality Weekly Report, 50(7), 120-125.

[3] Issa, S. N., \& Sharma, L. (2006). Epidemiology of osteoarthritis: an update. Current rheumatology reports, $8(1), 7-15$

[4] Buckwalter, J. A., Saltzman, C., \& Brown, T. (2004). The impact of osteoarthritis: implications for research. Clinical Orthopaedics and Related Research (427 Suppl.), S6-S15.

[5] Tarigan, T. J. E., Kasjmir, Y. I., Atmakusuma, D., Lydia, A., Bashiruddin, J., Kusumawijaya, K., \& Prihartono, J. (2009). The degree of radiographic abnormalities and postural instability in patients with knee osteoarthritis. Acta medica Indonesiana, 41(1), 15-19.

[6] Van der Pas, S., Castell, M. V., Cooper, C., Denkinger, M., Dennison, E. M., Edwards, M. H., Deeg, D. J. (2013). European project on osteoarthritis: design of a six-cohort study on the personal and societal burden of osteoarthritis in an older European population. Bmc Musculoskeletal Disorders, 14, 138. doi: 10.1186/1471-2474-14-138

[7] Wylde, V., Palmer, S., Learmonth, I. D., \& Dieppe, P. (2012). Somatosensory abnormalities in knee OA. Rheumatology (Oxford), 51(3), 535-543. doi: $10.1093 /$ rheumatology/ker343

[8] Hatfield, G., Hammond, C., \& Hunt, M. A. (2015). Clinical tests of balance in the knee osteoarthritis population: A systematic review. Osteoarthritis and Cartilage, 23, A374. doi: 10.1016/j.joca.2015.02.688

[9] Hinman, R. S., Bennell, K. L., Metcalf, B. R., \& Crossley, K. M. (2002). Balance impairments in individuals with symptomatic knee osteoarthritis: a comparison with matched controls using clinical tests. Rheumatology (Oxford), 41(12), 1388-1394

[10] Khalaj, N., Abu Osman, N. A., Mokhtar, A. H., Mehdikhani, M., \& Wan Abas, W. A. (2014). Balance and risk of fall in individuals with bilateral mild and moderate knee osteoarthritis. Plos One, 9(3), e92270. doi: 10.1371/journal.pone.0092270

[11] Dore, A. L., Golightly, Y. M., Mercer, V. S., Shi, X. A., Renner, J. B., Jordan, J. M., \& Nelson, A. E. (2015). Lower-extremity osteoarthritis and the risk of falls in a community-based longitudinal study of adults with and without osteoarthritis. Arthritis Care Res (Hoboken), 67(5), 633-639. doi: 10.1002/acr.22499

[12] Barbour, K. E., Stevens, J. A., Helmick, C. G., Luo, Y.-H., Murphy, L. B., Hootman, J. M., Sugerman, D. E. (2014). Falls and fall injuries among adults with arthritis - United States, 2012. MMWR: Morbidity \& Mortality Weekly Report, 63(17), 379-383.

[13] Fitzpatrick, R., Rogers, D. K., \& McCloskey, D. I. (1994). Stable human standing with lower-limb muscle afferents providing the only sensory input. The Journal of physiology, 480(Pt 2), 395-403.

[14] Zhang, S., \& Li, L. (2013). The differential effects of foot sole sensory on plantar pressure distribution between balance and gait. Gait \& Posture, 37(4), 532-535. doi: 10.1016/j.gaitpost.2012.09.012

[15] Kennedy, P. M., \& Inglis, J. T. (2002). Distribution and behaviour of glabrous cutaneous receptors in the human foot sole. The Journal of physiology, 538(Pt 3), 995-1002. doi: 10.1113/jphysiol.2001.013087

[16] Perry, S. D. (2006). Evaluation of age-related plantar-surface insensitivity and onset age of advanced insensitivity in older adults using vibratory and touch sensation tests. Neuroscience Letters, 392(1-2), 62-67. doi: http://dx.doi.org/10.1016/j.neulet.2005.08.060

[17] Hong, S. L., Manor, B., \& Li, L. (2007). Stance and sensory feedback influence on postural dynamics. Neuroscience Letters, 423(2), 104-108. doi: 10.1016/j.neulet.2007.06.043

[18] Lowrey, C. R., Strzalkowski, N. D., \& Bent, L. R. (2013). Cooling reduces the cutaneous afferent firing response to vibratory stimuli in glabrous skin of the human foot sole. Journal of Neurophysiology, 109(3), 839-850. doi: 10.1152/jn.00381.2012

[19] Orth, D., Davids, K., Wheat, J., Seifert, L., Liukkonen, J., Jaakkola, T., Kerr, G. (2013). The role of textured material in supporting perceptual-motor functions. Plos One, 8(4), e60349. doi: 10.1371/journal.pone.0060349

[20] Qiu, F., Cole, M. H., Davids, K. W., Hennig, E. M., Silburn, P. A., Netscher, H., \& Kerr, G. K. (2012). Enhanced somatosensory information decreases postural sway in older people. Gait \& Posture, 35(4), 630-635. doi: 10.1016/j.gaitpost.2011.12.013

[21] Roos, E. M., Herzog, W., Block, J. A., \& Bennell, K. L. (2011). Muscle weakness, afferent sensory dysfunction and exercise in knee osteoarthritis. Nature reviews. Rheumatology, 7(1), 57-63.

[22] Cavanaugh, J. T., Mercer, V. S., \& Stergiou, N. (2007). Approximate entropy detects the effect of a secondary cognitive task on postural control in healthy young adults: a methodological report. Journal of Neuroengineering and Rehabilitation, 4, 42. doi: 10.1186/1743-0003-4-42

[23] Wrisley, D. M., Stephens, M. J., Mosley, S., Wojnowski, A., Duffy, J., \& Burkard, R. (2007). Learning effects of repetitive administrations of the sensory organization test in healthy young adults. Arch Phys Med Rehabil, 88(8), 1049-1054. doi: 10.1016/j.apmr.2007.05.003

[24] Vanicek, N., Strike, S., McNaughton, L., \& Polman, R. (2009). Postural Responses to Dynamic Perturbations in Amputee Fallers Versus Nonfallers: A Comparative Study With Able-Bodied Subjects. Archives of Physical Medicine and Rehabilitation, 90(6), 1018-1025. doi: DOI 10.1016/j.apmr.2008.12.024

[25] Cohen, H., Heaton, L. G., Congdon, S. L., \& Jenkins, H. A. (1996). Changes in sensory organization test scores with age. Age and Ageing, 25(1), 39-44.

[26] Hatton, A. L., Dixon, J., Rome, K., Brauer, S. G., Williams, K., \& Kerr, G. (2016). The effects of prolonged wear of textured shoe insoles on gait, foot sensation and proprioception in people with multiple sclerosis: study protocol for a randomised controlled trial. Trials, 17(1), 208. doi: 10.1186/s13063-016-1337-x

[27] Priplata, A., Niemi, J., Salen, M., Harry, J., Lipsitz, L. A., \& Collins, J. J. (2002). Noise-enhanced human balance control. Physical Review Letters, 89(23), 238101. 
[28] Collins, J. J., Priplata, A. A., Gravelle, D. C., Niemi, J., Harry, J., \& Lipsitz, L. A. (2003). Noise-enhanced human sensorimotor function. IEEE engineering in medicine and biology magazine: the quarterly magazine of the Engineering in Medicine \& Biology Society, 22(2), 76-83.

[29] Priplata, A. A., Patritti, B. L., Niemi, J. B., Hughes, R., Gravelle, D. C., Lipsitz, L. A., . . . Collins, J. J. (2006). Noise-enhanced balance control in patients with diabetes and patients with stroke. Annals of Neurology, 59(1), 4-12. doi: 10.1002/ana.20670

[30] Hatton, A. L., Dixon, J., Rome, K., Newton, J. L., \& Martin, D. J. (2012). Altering gait by way of stimulation of the plantar surface of the foot: the immediate effect of wearing textured insoles in older fallers. Journal of Foot and Ankle Research, 5, 11. doi: 10.1186/1757-1146-5-11

[31] Qiu, F., Cole, M. H., Davids, K. W., Hennig, E. M., Silburn, P. A., Netscher, H., \& Kerr, G. K. (2013). Effects of textured insoles on balance in people with Parkinson's disease. Plos One, 8(12), e83309. doi: 10.1371/journal.pone.0083309

[32] Palluel, E., Olivier, I., \& Nougier, V. (2009). The lasting effects of spike insoles on postural control in the elderly. Behavioral Neuroscience, 123(5), 1141-1147. doi: 10.1037/a0017115

[33] Hassan, B. S., Mockett, S., \& Doherty, M. (2001). Static postural sway, proprioception, and maximal voluntary quadriceps contraction in patients with knee osteoarthritis and normal control subjects. Annals of the Rheumatic Diseases, 60(6), 612-618.

[34] Clavet, H., Hébert, P. C., Fergusson, D., Doucette, S., \& Trudel, G. (2008). Joint contracture following prolonged stay in the intensive care unit. CMAJ: Canadian Medical Association Journal = Journal De L'association Medicale Canadienne, 178(6), 691-697. doi: 10.1503/cmaj.071056

[35] Gauchard, G. C., Gangloff, P., Jeandel, C., \& Perrin, P. P. (2003). Physical activity improves gaze and posture control in the elderly. Neuroscience Research, 45(4), 409-417. doi: http://dx.doi.org/10.1016/S0168-0102(03)00008-7

[36] Allum, J. H., \& Shepard, N. T. (1999). An overview of the clinical use of dynamic posturography in the differential diagnosis of balance disorders. Journal of vestibular research: equilibrium \& orientation, 9(4), 223-252.

[37] Takacs, J., Carpenter, M. G., Garland, S. J., \& Hunt, M. A. (2013). The Role of Neuromuscular Changes in Aging and Knee Osteoarthritis on Dynamic Postural Control. Aging and disease, 4(2), 84-99.

[38] Hirata, R. P., Ervilha, U. F., Arendt-Nielsen, L., \& Graven-Nielsen, T. (2011). Experimental muscle pain challenges the postural stability during quiet stance and unexpected posture perturbation. The journal of pain: official journal of the American Pain Society, 12(8), 911-919. doi: 10.1016/j.jpain.2011.02.356

[39] Jones, K. D., King, L. A., Mist, S. D., Bennett, R. M., \& Horak, F. B. (2011). Postural control deficits in people with fibromyalgia: a pilot study. Arthritis Research \& Therapy, 13(4).

[40] Cheng, Y.-Y., Chen, P.-Y., Hsieh, W.-L., Cheen, J.-R., \& Kao, C.-L. (2012). Correlation of the composite equilibrium score of computerized dynamic posturography and clinical balance tests. Journal of Clinical Gerontology and Geriatrics, 3(2), 77-81. doi: https://doi.org/10.1016/j.jcgg.2012.04.004. 